F. Maloberti: "Architectures and Design Methodologies for Very Low Power and Power EffectiveA/D Converters"; IEEE North-East Workshop on Circuits and Systems, Plenary Talk, 2006, Gatineau, 18-21 June 2006, pp. 77-80.

(C)20xx IEEE. Personal use of this material is permitted. However, permission to reprint/republish this material for advertising or promotional purposes or for creating new collective works for resale or redistribution to servers or lists, or to reuse any copyrighted component of this work in other works must be obtained from the IEEE. 


\title{
Architectures and Design Methodologies for Very Low Power and Power Effective A/D Converters
}

\author{
Franco Maloberti \\ Department of Electronics, University of Pavia \\ Via Ferrata 1, 27100 Pavia Italy \\ franco.maloberti@unipv.it
}

\begin{abstract}
The main goal of portable applications is obtaining data conversion with very low power consumption while maintaining acceptable resolution and linearity. This paper presents various design methods for achieving figure of merit of $1 \mathrm{pJ}$ conv or less, usable other than sigma-delta architectures in pipeline and successive approximation algorithms. Results of state-of-the art designs are presented.

Index Terms-Low-power, Figure of merit (FoM).
\end{abstract}

\section{INTRODUCTION}

The technology advancements and the electronics market evolution more and more favor applications with nomadic features. The mobility request involves performing complex functions with a limited power refueling. Other emerging needs impose an autonomous operation that means that no power is specifically provided and the system must have the capability to acquire the power that needs and modulate its activity depending on the available power budget.

Since for nomadic electronics the most relevant feature is the optimum trade-off between power and performance, minimum power and its aware use are the major design concern. New research topics are ultra-low power analog conditioning, ultra-low power data conversion and power aware digital design that involves re-considering the design of basic digital cells and the power optimization of algorithms.

For the design of data converter the effective use of power is measured by the figure of merit $(F o M)$ defined as

$$
F_{O M}=\frac{P}{2^{E N O B} \cdot 2 \cdot f_{B}}
$$

where $E N O B$ is the equivalent number of bits and $f_{B}$ is the maximum bandwidth of the input signal, equal to $f_{s} / 2$ for Nyquist-rate converters and for oversampling architectures $f_{s} /(2 \cdot O S R)$ (where $O S R$ is the oversampling ratio).

The use of the FoM parameter is not a solid way to asses the power effectiveness: the FoM depends on technology and the frequency range of operation: very low speeds enable using power effective solutions and obtain very good FoM. On the contrary, achieving very high resolutions and large bandwidths is more demanding in term of power use. As a matter of facts, the more suitable data converter architecture mainly depends on the signal band. Signals with very low bandwidth as the ones generated by biologic systems enable low sampling frequency that, by turns, makes it possible to use many clock periods for implementing the conversion algorithm. This possibility can be exploited to optimize the power consumption. When the signal band is large the limit comes from the need of using high speed electronics that, for $C M O S$ implementations, almost quadruples the power for every doubling of the operational bandwidth. Nevertheless, obtaining a FoM in the range of $1 p J$-conv or less is a good sign of an effective use of power.

This paper revisits recently proposed design techniques with focus the obtained power effectiveness. The proposed techniques are used in sigma-delta architectures. However, the same methods can be conveniently employed in other like the successive approximation or the pipeline architecture.

\section{Sigma-Delta ARChitectures}

The sigma-delta $(\Sigma \Delta)$ technique was used for obtaining high-resolution, low-bandwidth analog-to-digital converters. Now, the oversampling method is also used for low-power high-bandwidth systems operating at medium-resolution that employ a small $O S R$ and exploit the benefit of using analog components with limited accuracy and low voltage for an integration in digital CMOS processes.

The low power goal can be obtained by a proper choice of three design parameters: the oversampling ratio, the order of the modulator and the number of bits of the quantization. For this, observe that:

- The resolution increases with the sampling frequency but augments the need of high bandwidth and slew-rate in the used OTAs, thus affecting the power consumption.

- High-order obtains a better noise shaping but requires a large number of OTAs (and, consequently consumes power); also, stability in high-order modulators is ensured by feedback branches that, limiting the oversampling benefits, require more power.

- Multi-bit quantizers increases the resolution but using the multi-bit $D A C$ is source of harmonic distortion.

Since the latter limit can be fixed by using dynamic element matching $(D E M)$ that transforms the mismatch between unity elements into noise, a first design strategy is to identify the best choice of modulator order and number of bits of the $A D C$.

Another design direction is the minimization of the number of OTA. This can be obtained by sharing functions or, as it will be shown shortly, by using a single op-amp for realizing high order transfer functions.

Since the reduction of the clock frequency by a factor $k$ diminishes the power consumption of the OTA by $k^{\alpha}$, where 


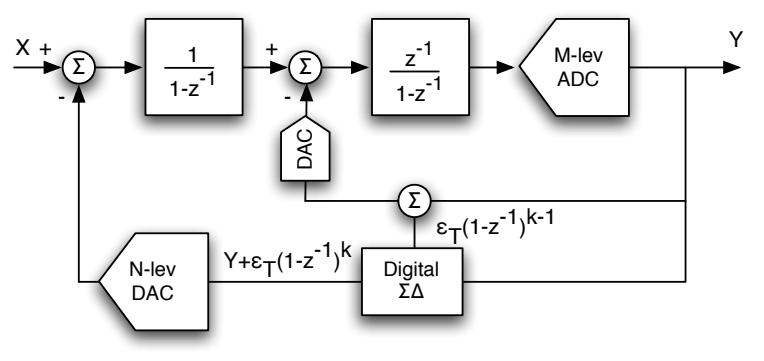

Fig. 1. Second order $\Sigma \Delta$ Modulator with reduced DAC resolution.

$\alpha \simeq 2$, the clock used for the operation of the circuit must be as low as possible. Therefore, time interleaved architectures that reduce the clock frequency in the paths obtains a total power that is lower than single path conventional solutions.

Obviously, the power consumption is also reduced by a careful design of basic components (OTAs and comparators) and a proper choice of architectures. For example, limiting the voltage swing of the integrators benefits the slew-rate requirements; using modulators that consent low gain enables simple OTA schemes; limiting the swing at the input of the $A D C$ reduces the number of required comparators.

\section{DAC RESOLUTION REDUCTION}

Since $k$-comparators flash grants $\log _{2}(k-1)$ additional bits either for a Nyquist-rate or a $\Sigma \Delta$, increasing by $2^{2.5} \simeq 6$ the number of comparators in second-order modulator is equivalent to doubling the $O S R$. Typically the power of extra comparators is lower than the one necessary to double the bandwidth and the slew rate of the two OTAs as a comparator consumes from $1 / 20$ to $1 / 40$ the power of an OTA with comparable speed. However, the request of an effective DEM limits the practical maximum of the comparators.

The DAC can use less levels than the ADC thanks to the Leslie-Sing [1] approach or the truncation and cancellation method that, as shown in Fig. 1, uses a digital $\Sigma \Delta$ that reduces the number of bits by shaping the truncation error

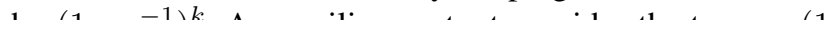

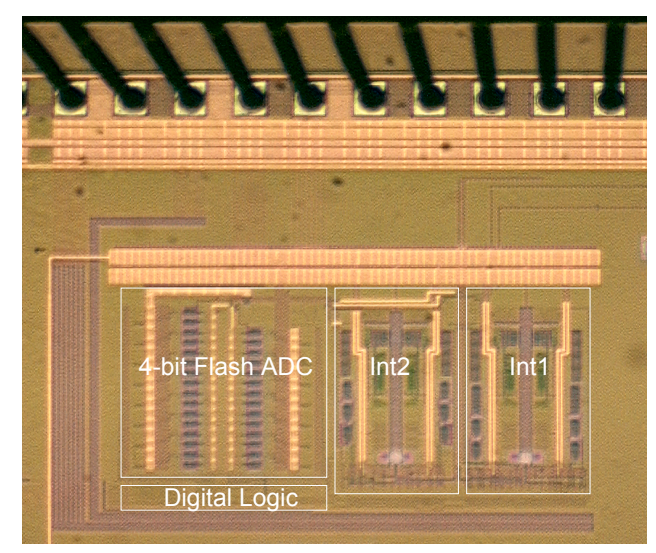

Fig. 2. Microphotograph of the $\Sigma \Delta$ Modulator with reduced DACs resoluton. $\left.z^{-1}\right)^{k-1}$ that cancels the effect of the shaped truncation noise injected by the input $D A C$. Indeed, as it can be verified by inspection, the shaped truncation noise $\epsilon_{T}\left(1-z^{-1}\right)^{k}$ passed through the first integrator equals the auxiliary output.

The technique can also be applied to reduce the resolution of second DAC. In this case the canceling term is directly subtracted in the digital domain. The method was used for obtaining 10-bit employs a 4-bit ADC and DACs with 3 level and 5 levels, enabling $1 \mathrm{MHz}$ signal band with $2.1 \mathrm{~mW}$ [2].

\section{REDUCTION OF THE NUMBER OF OTAS}

The order of a $\Sigma \Delta$ converter determines the number of OTA used by the architecture. However, the number of $O T A$ s can be reduced by sharing functions. With switched capacitor circuit the double sampling technique or the opamp sharing reduce the op-amp count but the used power increases because of the augmented slewing requirements. The power benefit is about $30 \%$.

A more effective approach is the one proposed in [3] as an extension of the the technique described in [4]. Consider Fig. 3 showing a quite general $\Sigma \Delta$ modulator. It is the cascade of integrator with distributed feedbacks and no feed-forwards.

Since the feedback path at the input of the $i$-th stage can be moved to the input of the previous $(i-1)$-th stage by dividing its contribution by $H_{i-1}(z)$, it is possible to remove, by successive iterations all the internal feedbacks for obtaining the scheme of Fig. 4. Since the feedback from the digital output uses digital-to-analog conversion, the signal processing is performed in the digital domain before the $D A C$. The coefficient $k_{T}$ is

$$
k_{T}=k_{1}+\frac{k_{2}}{H_{1}(z)}+\cdots+\frac{k_{n}}{\prod_{1}^{n-1} H_{i}(z)}
$$

The cascade of analog blocks $H_{1}(z), \cdots, H_{n}(z)$, can be realized with a minimum number of operational amplifiers. Since sigma delta modulators use integrators it results

$$
H_{T}(z)=\frac{z_{p}}{\left(1-z^{-1}\right)^{n}}
$$

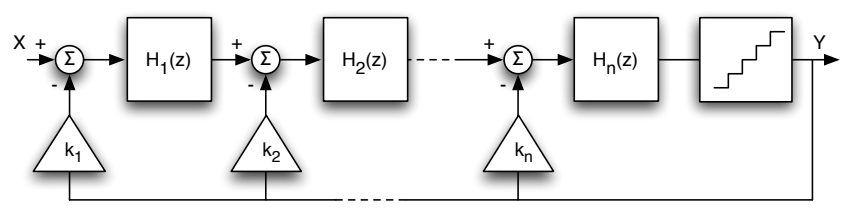

Fig. 3. Generic Scheme of a $\Sigma \Delta$ Modulator.

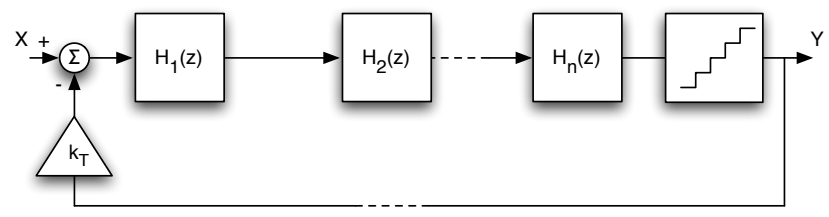

Fig. 4. Modified Scheme of a $\Sigma \Delta$ Modulator. 


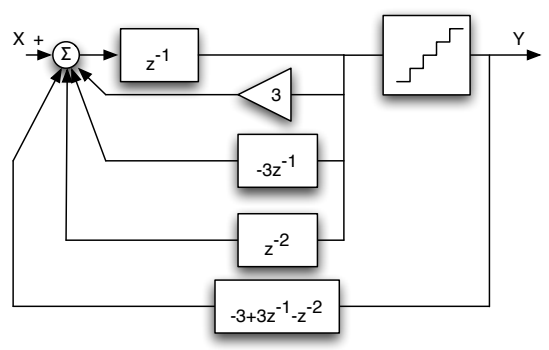

Fig. 5. Single OTA implementation of a third order $\Sigma \Delta$.

A third order scheme is realized by block diagram of Fig. 5. The filter $P_{n}(z)$ is given by

$$
P_{3}(z)=3-3 z^{-1}+z^{-2}
$$

The filter $P_{n}(z)$ is achieved in a passive way for using only one $O T A$.

The scheme used for WCDMA (signal bandwidth 1.92 $M H z$ and $S N D R=60 \mathrm{~dB}$ ) with 12-level $A D C$ enables oversampling $O S R=10$. Since the double sampling technique leads to a $19.2 \mathrm{MHz}$ clock frequency the $40 \mathrm{MHz} f_{T}$ opamp uses only $0.4 \mathrm{~mA}$. If the $A D C$ consumes $0.2 \mathrm{~mA}$ and the digital part $0.1 \mathrm{~mA}$ the power consumption is $0.85 \mathrm{~mW}$ at $1.2 \mathrm{~V}$ corresponding to $F o M=0.22 \mathrm{pJ} /$ conv-level.

\section{Time Interleaved Technique}

The time-interleaved (TI) technique is attractive for highspeed applications as the oversampling rate $(O S R)$ increases without requiring to speed-up the analog blocks. However, the recursive operation of $\Sigma \Delta$ modulators, which is not featured by Nyquist rate interleaved converters, complicates the transformation of $\Sigma \Delta$ modulators into TI equivalent structures. The TI $\Sigma \Delta$ modulators proposed in the literature conceptually works well but the so called quantizer domino and channel mismatch limit the implementations. The quantizer domino occurs when a certain quantizer output is connected to another quantizer input via an analog block without a delay. Because of the domino effect timeinterleaved modulators can use only two paths and double sampling.

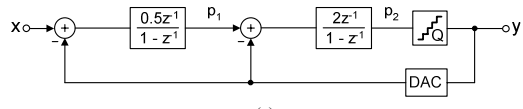

(a)

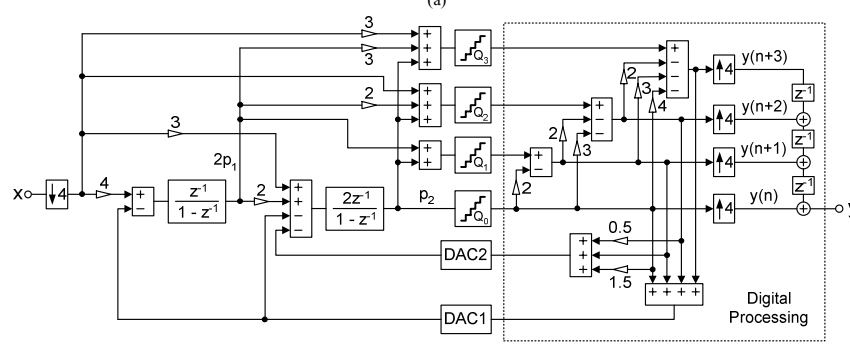

(b)

Fig. 1 Second order sigma-delta modulator. (a) Conventional single-path. (b) Proposed 4-path TI

Fig. 6. a) Second Order sigma-delta modulator. b) The 4-path time

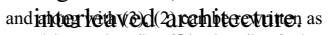
$p_{2}(n)=p_{2}(n-4)+2\left[4 p_{1}(n-4)+3 x(n-4)\right.$

$$
\left.-0.5 y(n-2)-y(n-3)-1.5 y(n-4)-\sum^{4} y(n-i)\right]
$$

The first and second integrator, shown in Fig. 1(b) directly implements (4) and (5). Since, the quantizer processes $p_{2}$, it is necessary to estimate the $(n+r)$-th time slot outputs of $p_{2}$ for $r=1,2,3$. This expanded into $r$-consecutive time slots is given by 3. SIMULATION RESULTS

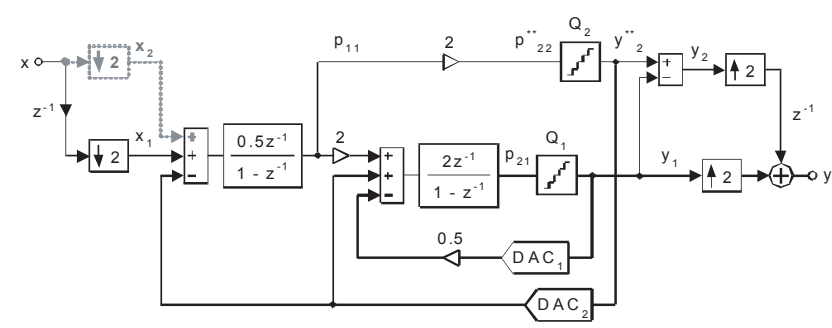

Fig. 7. Two-path TI second-order sigma-delta modulator.

The problem is overcame in [5] that enables achieving a 4-path TI modulator. The basic idea, embedded in the scheme of Fig. 6, is removing from three of the four paths the recursive signal, as it is not available, for obtaining incomplete quantized outputs. A suitable signal processing recovers in the digital domain the missing parts and obtains the four sequential outputs. The second order modulator of Fig. 6 uses 2 OTAs for the main path. Moreover, incomplete signals that are only additions of terms require using one OTA for each of the three other paths.

The number of bits of the flashes increases along the paths for accommodating the enlarging swing caused by the incomplete signals; therefore, if the main $D A C$ uses 2-bit the other DACs require 3, 4, 5-bit respectively. In summary, the architecture uses 5 OTA and 56 comparators that run at a quarter of the clock frequency while the single path counterpart should use 2 OTA and 3 comparators running at the full speed. The estimation of power reduction is

The 4-path architecture is suitable for signal bands in the $10-20 \mathrm{MHz}$ range, as it significantly reduces the power consumption and, perhaps, makes it possible oversampling ratios that the technology is not able to obtain. If, for example, the clock is $240 \mathrm{MHz}$ clock (that means an effective sampling rate of $960 \mathrm{MHz}$ ) and $O S R=32$ the $S N R$ of a 3 bit modulator becomes $62 \mathrm{~dB}$ for a signal band of $10 \mathrm{MHz}$. The estimated power consumption is $3 \mathrm{~mW}$ for the OTAs and $40 \mu \mathrm{W}$ for the comparators, leading to less than $28 \mathrm{~mW}$ (including the power of the digital section) corresponding to FoM $=0.93 \mathrm{pJ} /$ conv-level which is a good number for 15 $\mathrm{MHz}$ bandwidth.

For low-voltage applications and small signal bandwidth like the one required by $A D S L$ a two-path architecture is a better design choice. The architecture shown in Fig. 7 uses a 5-level quantizers and a clock rate $f_{s}=66 \mathrm{MHz}$ for obtaining, thanks to the $O S R$ doubling, an $O S R=60$ at a signal bandwidth of $1.1 \mathrm{MHz}$. The design discussed in [6], whose chip microphotograph is shown in Fig. 8, uses OTA's $D C$ gain equal to $70 \mathrm{~dB}$ and $50 \mathrm{~dB}$ for the first and the second integrator and sampling capacitors equal to $1.6 \mathrm{pF}$ and $0.8 \mathrm{pF}$. The experimental results show a peak $S N D R$ equal to $76 d B$ and $D R=85 \mathrm{~dB}$. The power consumption with supply voltage Therefore, the modulator outputs for time slot $n, n+1, n+21.8 \mathrm{~V}$ is $5.4 \mathrm{~mA}$ giving a $\mathrm{FoM}=0.47 \mathrm{pJ} / \mathrm{conv}$-level. and $n+3$ can be obtained by processing the quantizer
outputs in the digital domain. That is, $y(n+1)$ can be outputs in the digital domain. That is, $y(n+1)$ can be
obtained from $Q_{1}$ and $y(n) ; y(n+2)$ from $Q_{2}, y(n)$ and obtained from $Q_{1}$ and $y(n) ; y(n+2)$ from $Q_{2}, y(n)$
$y(n+1) ; y(n+3)$ from $Q_{3}, y(n), y(n+1)$ and $y(n+2)$. 


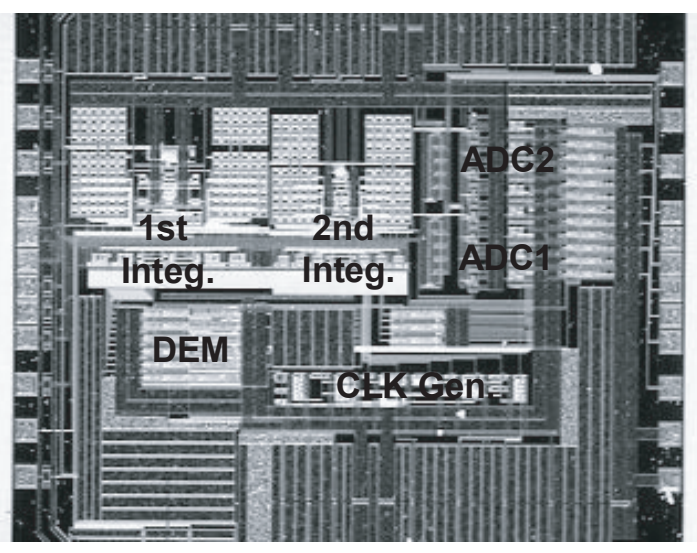

Fig. 8. Microphotograph of a TI interleaved modulator for ADSL.

\section{OTA SWING REDUCTION}

Another technique used for reducing the power consumption minimizes the OTA output swing: the slew-rate requirements are relaxed, the power consumed for charging capacitors is limited and, also, makes it possible using power effective OTAs like single-stage telescopic schemes.

The op-amp swing is reduced by using analog feedforward $(A F F)$. The injection of the input signal into an op-amp almost reduces by the same amount the swing of the previous integrator. Therefore, the swing goes down to the quantization noise that, for multi-bit architectures, is a fraction of the maximum input. The method works well for all the integrators out of the last one as the analog addition would require, in front of the multi-bit flash an extra OTA.

The solution proposed by [7] performs the last addition in the digital domain as Fig. 9 shows. The subtraction requires a second $A D C$ but, thanks to the swing reduction the total number of comparators goes down instead of increasing.

As shown in Fig. 9 the output of the auxiliary flash that provides the digital feed-forward $(D F F)$ signal brings about quantization noise which effect is cancelled out by the signal injection at the input of the second integrator. The processing of the used $K$ block cancels the contribution of the $D F F$ being $K=\frac{z^{-1}-1}{a_{2}}$, that is a simple operation to be performed in the analog domain.

The number of bits of the two quantizers are, for the full

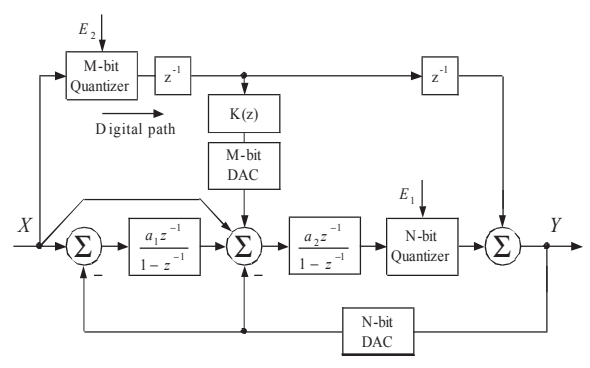

Fig. 9. Scheme of the OTA swing reduction method.

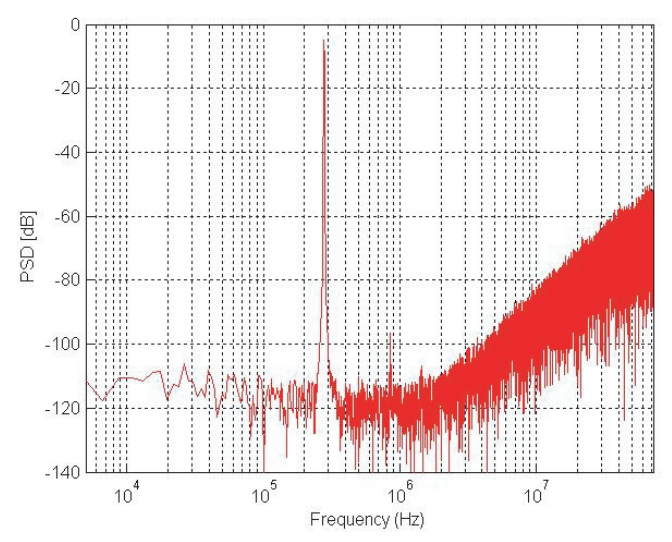

Fig. 10. Spectrum of the modulator with OTA swing reduction.

$\pm V_{\text {ref }}$ range, $N=4$ and $M=3$. However, the swing reduction determines an operation range of the second OTA that requires only 5 comparators. As a result, the $S N R$ is the one of a 4-bit $\Sigma \Delta$ modulator with total $7+5$ comparators.

Simulation results show that the 3-bit auxiliary flash reduces the swing of the second OTA down to $\pm 0.32 V_{\text {ref }}$; therefore, in addition to a reduced number of quantization level the op-amp power also diminishes. The swing at the output of the first $O T A$ is low too: $\pm 0.15 V_{\text {ref }}$.

The circuit implementation for meeting the ADSL+ specifications uses a single poly 6-metal $0.18 \mu \mathrm{m}$ CMOS technology and consumes $5.1 \mathrm{~mW}$ in the analog section and $8.7 \mathrm{~mW}$ in the digital part. The relatively high digital power is due to a $2.5 \mathrm{~V}$ digital supply for having the operation of the logic properly. Therefore, the figure of merit $F o M=$ $0.48 \mathrm{pJ} / \mathrm{conv}$-level could be possibly reduced by optimizing the digital design. Figure 10 shows the measured 65536points power spectrum for a $280 \mathrm{kHz},-4 d B_{F S}$ differential sinusoidal input. The peak $S N R$ is $82.4 d B$ and the peak $S N D R$ is $77.6 d B$ fully complaining with the $A D S L+$ specifications.

\section{REFERENCES}

[1] Leslie, T.C., Singh, B.: "Sigma-delta modulators with multibit quantising elements and single-bit feedback", IEE Proceedings G Circuits, Devices and Systems, Volume: 139, Issue: 3, June 1992, Pages: 356 362.

[2] Yu, Y., Maloberti, F.: "A Low-Power Multi-Bit $\Sigma \Delta$ Modulator in 90-nm Digital CMOS Without DEM", IEEE Journal of Solid-State Circuits, 2005, December, vol. 40, Pages: 2428-2436.

[3] Della Fiore, C., Maloberti, F.: "Design of $\Sigma \Delta$ Modulators with Reduced Number of Operational Amplifiers" ECCTD, Proceedings, 2005.

[4] Koh, J., Choi, Y., Gomez, G.: A 66dB DR 1.2V 1.2mW SingleAmplifier Double-Sampling 2nd-order $\Sigma \Delta$ ADC for WCDMA in $90 \mathrm{~nm}$ CMOS, IEEE-ISSCC 2005, 9.3, pp.170-171.

[5] Kye-Shin Lee, Choi, Y., Maloberti, F.: "Domino Free 4-path TimeInterleaved Second Order Sigma-Delta Modulator", Analog Integrated Circuits and Signal Processing, Volume 43,2005, Pages 225-236.

[6] Kye-Shin Lee, Sunwoo Kwon, Maloberti, F.: A 1.1 MHz Signal-Band 2-Channel Time-Interleaved Multi-bit $\Sigma \Delta$ Modulator with 80dB SNR $85 \mathrm{~dB}$ DR and $5.4 \mathrm{~mW}$ Power Consumption for ADSL, IEEE-ISSCC 2006

[7] Sunwoo Kwon, Maloberti, F.: A $2.2 \mathrm{MHz}$ Signal Band Multi-bit $\Sigma \Delta$ Modulator with $82 \mathrm{~dB}$ SNR and $86 \mathrm{~dB}$ DR for ADSL2+, IEEE-ISSCC 2006. 